\title{
Caracterização epidemiológica da mortalidade por Alzheimer no Brasil entre 2010 a
}

\section{9}

Epidemiological characterization of Alzheimer mortality in Brazil from 2010 to 2019

Caracterización epidemiológica de la mortalidade por Alzheimer en Brasil entre 2010 a 2019

Recebido: 11/08/2021 | Revisado: 11/08/2021 | Aceito: 21/08/2021 | Publicado: 23/08/2021

Dalton Ferreira Matos

ORCID: https://orcid.org/0000-0002-6188-7536 Universidade Federal de Sergipe, Brasil

E-mail: daltonmatosgn@gmail.com

Wandklebson Silva da Paz

ORCID: https://orcid.org/0000-0002-2898-2646

Universidade Federal de Pernambuco, Brasil

E-mail: wandklebson.paz@gmail.com

Allan Bruno Alves de Sousa Santos

ORCID: https://orcid.org/0000-0001-6412-7164

Faculdade de Educação São Francisco, Brasil

E-mail: Abass@ faesf.com.br

Mikaelle Alves Silva

ORCID: https://orcid.org/0000-0002-9336-1491

Universidade Federal do Espírito Santo, Brasil

E-mail: farm.mikaelle@gmail.com

Samuel Felício de Oliveira

ORCID: https://orcid.org/0000-0001-6482-4808

Centro Universitário Alfredo Nasser, Brasil

E-mail: felicio.samuel306@gmail.com

Carla Conrado Barbosa Leite

ORCID: https://orcid.org/0000-0002-7033-3682

Faculdade da Polícia Militar, Brasil

E-mail: carlaconradobl@gmail.com

Millena Carla Rosa Silva

ORCID: https://orcid.org/0000-0001-8065-4781

Centro Universitário Alfredo Nasser, Brasi

E-mail: millenacarlla@gmail.com

André Cardoso Tavares

ORCID: https://orcid.org/0000-0002-8413-3868

Centro Universitário Unifacid, Brasil

E-mail: andrebiomed16@gmail.com

Debora Kallyne Da Silva Oliveira

ORCID: https://orcid.org/0000-0003-4574-7741

Centro Universitário Alfredo Nasser, Brasil

E-mail: deborakallynne47@icloud.com

Mayara Martins Teles

ORCID: https://orcid.org/0000-0002-1023-2863

Centro Universitário Alfredo Nasser, Brasil

E-mail: mayara.teles21@gmail.com

Geovanna Magalhaes da Silva

ORCID: https://orcid.org/0000-0002-1522-554X

Centro universitário Alfredo Nasser, Brasil

E-mail: geomagalhaesss@gmail.com

Rafael dos Santos Balbino

ORCID: https://orcid.org/0000-0001-6375-2021

Instituto Federal de Alagoas, Brasil

E-mail: rafael.balbino@ifal.edu.br

Mariana Marques Pinto

ORCID: https://orcid.org/0000-0003-0806-7958

Centro Universitário Alfredo Nasser, Brasil

E-mail: marianamarques02002@gmail.com

Robert Lincoln Barros Melo

ORCID: https://orcid.org/0000-0002-3430-4411

Fundação Oswaldo Cruz, Brasil

E-mail: robert.lincoln.b@gmail.com 


\title{
Resumo
}

O Alzheimer é uma doença do cérebro que está relacionada à idade do paciente, na qual a compreensão de sua progressão pode ser favorável para prevenir a doença através de uma intervenção precoce. As diferentes taxas de mortalidade nas grandes regiões do país apresentam desigualdades no desenvolvimento socioeconômico, necessitando de políticas públicas mais eficientes. Com isso está pesquisa é um estudo descritivo transversal epidemiológico, com o intuito de verificar a mortalidade por Doença de Alzheimer no Brasil durante os anos de 2010 a 2019, empregando dados de base populacional, de acordo a Secretaria de Vigilância em Saúde do Ministério da Saúde (SVS/MS) a partir dos bancos de dados do Sistema de Informação de Agravos de Notificação (SINAN). Onde no Brasil as características sociodemográficas predominantes foram: sexo feminino $(64,54 \%)$, faixa etária $\geq 80(74,99 \%)$; pessoas brancas $(73,80 \%)$ e pessoas viúvas $(50,66 \%)$, no entanto, alguns dados foram ignorados $(9,42 \%)$ em que não se sabe o gênero, a faixa etária, a raça e o estado civil de alguns pacientes. Dessa forma, esse trabalho enfatiza a necessidade de investimentos em saúde pública, pois só assim os pacientes que possuem a doença de Alzheimer poderão ter atenção ao atendimento e adesão ao tratamento referente a essa doença.

Palavras-chave: Epidemiologia; Doença de Alzheimer; Cérebro.

\begin{abstract}
Alzheimer is a brain disease that is linked to the patient's age, in which an understanding of its progression can be favorable to prevent the disease through early intervention. The different mortality rates in the large regions of the country present inequalities in socioeconomic development, requiring more efficient public policies. Hence, this research is a descriptive cross-sectional epidemiological study, with the aim of verifying mortality from Alzheimer's Disease in Brazil during the years 2010 to 2019, using population-based data, according to the Ministry's Health Surveillance Secretariat (SVS/MS) from the databases of the Notifiable Diseases Information System (SINAN), where in Brazil the predominant sociodemographic characteristics were: female (64.54\%), age group $\geq 80$ (74.99\%); white people $(73.80 \%)$ and widowed people (50.66\%). However, some data were ignored $(9.42 \%)$, in which the gender, age, race and marital status of some patients are unknown. Thus, this work emphasizes the need for investments in public health, as this is the only way in which patients who have Alzheimer's disease will be able to pay attention to care and adherence to treatment related to this disease.
\end{abstract}

Keywords: Epidemiology; Alzheimer's disease; Brain.

\section{Resumen}

El Alzheimer es una enfermedad cerebral relacionada con la edad del paciente, en la que la comprensión de su progresión puede ser favorable para prevenir la enfermedad a través de una intervención temprana. Las diferentes tasas de mortalidad en las grandes regiones del país presentan desigualdades en el desarrollo socioeconómico, lo que requiere políticas públicas más eficientes. Esta investigación es un estudio descriptivo epidemiológico transversal, con el objetivo de verificar la mortalidad por enfermedad de Alzheimer en Brasil durante los años 2010 a 2019, utilizando datos poblacionales, Según la Secretaría de Vigilancia Sanitaria del Ministerio de Salud (SVS/MS) de las bases de datos del Sistema de Datos de Notificación (SINAN). Donde las características sociodemográficas predominantes en Brasil fueron: sexo femenino (64,54\%), grupo de edad 80 (74,99\%); personas blancas $(73,80 \%)$ y viudas $(50,66 \%)$, sin embargo, algunos datos fueron ignorados $(9,42 \%)$ en los que se desconoce el sexo, edad, raza y estado civil de algunos pacientes. Así, este trabajo enfatiza la necesidad de inversiones en salud pública, porque solo entonces los pacientes con enfermedad de Alzheimer podrán prestar atención a la atención y adherencia al tratamiento relacionado con esta enfermedad.

Palabras clave: Epidemiología; Alzheimer; Cerebro. 


\section{Introdução}

O Alzheimer é uma doença do cérebro que está relacionada à idade do paciente na qual a compreensão de sua progressão pode ser favorável para prevenir a doença através de uma intervenção precoce (Yang et al., 2010). Long e Holtzman (2019) retratam que os avanços ao longo dos anos a respeito da doença de Alzheimer possuem algumas limitações como por exemplo, uma terapia que seja totalmente eficaz em seres humanos.

Tendo em vista que com o aumento da população global a expectativa de vida vem aumentando de forma exponencial ao longo das décadas, as doenças que se referem à terceira idade vêm demonstrando grande incidência, sendo uma delas o Alzheimer, considerada uma patologia cognitiva de maior prevalência em idosos (Magalhaes et al., 2020).

Em um relatório intitulado "Dementia a Public Health Priority" da Organização Mundial da Saúde de 2012, retrata que cerca de 35,5 milhões de pessoas vivem com demência e esse número irá dobrar em 2030 e poderá triplicar até 2050 . A demência tem um grande impacto na vida da família, pois seus membros são os que desempenham o papel principal no cuidado do paciente (OMS, 2012).

As implementações de novas metodologias para verificar a prevalência de Alzheimer a nível populacional, pode-se mostrar vantajoso para avaliar as manifestações patológicas, diferentes recursos tecnológicos e difundir planejamentos em saúde (Favaretto et al., 2021). As diferentes taxas de mortalidade nas grandes regiões do país apresentam desigualdades no desenvolvimento socioeconômico necessitando de políticas públicas mais eficientes no âmbito nacional com o intuito de diminuir a taxa de mortalidade nessas regiões (Pereira et al., 2021).

Com isso, analisar as notificações de mortes por Alzheimer no Brasil, pode fornecer melhor compreensão sobre a dinâmica da doença frente à população Brasileira. Além disso, são muito excassos os estudos relacionados à epidemiologia dessa doença no Brasil. Por conseguinte, o objetivo deste estudo é caracterizar o perfil epidemiológico e analisar a tendência temporal da mortalidade por Alzheimer no Brasil entre os anos de 2010 a 2019, empregando dados de base populacional.

\section{Metodologia}

\subsection{Tipo e área de estudo}

Foi projetado um estudo descrito transversal com o objetivo de descrever a mortalidade causada por Alzheimer no Brasil nos últimos 20 anos. O Brasil possui uma população de aproximadamente 210 milhões de habitantes segundo a estimativa intercensitária do IBGE em 2019. É dividido político e administrativamente em 27 unidades federativas (UF) (26 estados e um Distrito Federal) e 5,570 municípios. Para fins políticos e operacionais, as unidades da federação são agrupadas em cinco regiões (Norte, Nordeste, Sudeste, Sul e Centro-Oeste) com distintas características geográficas, econômicas e culturais (IBGE, 2020).

\subsection{Fonte de dados}

Os dados referentes aos óbitos por local de residência foram obtidos do Sistema de Informações sobre Mortalidade (SIM) do Ministério da Saúde. Os dados do SIM são de domínio público e podem ser obtidos a partir do site do Departamento de Informática do Sistema Único de Saúde (DATASUS). Os dados populacionais para o período de 2001 a 2019 foram obtidos do Instituto Brasileiro de Geografia e Estatística (IBGE), com base em dados oriundos do censo da população nacional em 2000 e 2010 e estimativas oficiais para os anos intercensitários (IBGE, 2020).

\subsection{Análise dos dados}

Primeiramente foi realizada a caracterização epidemiológica descritiva dos óbitos por Alzheimer, considerando as variáveis disponíveis na declaração de óbito. Com isso, foi feita a categorização das variáveis e quantificação dos casos a partir 
das seguintes variáveis sociodemográficas: região, sexo, faixa etária, raça/cor e estado civil. Adicionalmente, foram calculadas as taxas de mortalidade por Alzheimer por ano a nível de Brasil e calculadas as taxas médias de mortalidade por região brasileiras. Com os dados adquiridos sobre a doença foram armazenados através do programa Microsoft Word Excel 2019, onde foram elaboradas as tabelas e gráficos deste trabalho.

\section{Resultados e Discussão}

Entre os anos de 2010 a 2019, o SIM registrou 164.976 óbitos nas cinco regiões brasileiras. Dessas, a região Sudeste foi a que obteve o maior número de mortes por Alzheimer com 90.288 (54,72\%). No entanto, a região com maior taxa de mortalidade foi a região Sul, seguido da região Nordeste. Vale destacar que essas duas regiões apresentaram taxa de mortalidade superior a taxa brasileira. Por fim, as regiões Norte e Nordeste apresentaram os menores números de óbitos bem como menores taxas de mortalidade (Tabela).

Tabela 1. Caracterização dos óbitos por Alzheimer segundo de acordo com as 5 regiões brasileiras entre 2010 a 2019.

\begin{tabular}{llll}
\hline Regiões brasileiras & $\mathbf{N}^{\mathbf{o}}$ absoluto de casos & Frequência (\%) & $\begin{array}{l}\text { Taxa de média } \\
\text { de mortalidade }\end{array}$ \\
\hline Região Norte & 3.828 & 2,32 & 1,21 \\
Região Nordeste & 27.677 & 16,77 & 2,61 \\
Região Sudeste & 90.288 & 54,72 & 5,62 \\
Região Sul & 33.495 & 20,30 & 6,12 \\
Região Centro-Oeste & 9.688 & 5,87 & 3,45 \\
Total & 164.976 & 100,00 & 4,32 \\
\hline
\end{tabular}

Fonte: Autores.

Em um estudo realizado por Santos et al (2019), onde os pesquisadores verificaram a morbimortalidade de indivíduos hospitalizados no Brasil durante os anos de 2008 a 2018, mostraram que a região Sudeste teve o maior número de obtidos durantes os 10 anos, tendo similaridade com outros pesquisadores (Ferreira et al., 2016; Vidor et al., 2019).

Outro estudo de cunho estadual é o trabalho de Teixeira et al., (2012) onde os pesquisadores retratam que entre 2000 e 2006, no estado do Rio de Janeiro, teve um total e 2.992 óbitos, com uma maior prevalência no sexo feminino (65\%) da taxa de mortalidade nesse estado, mas devemos levar em consideração que na literatura cientifica a maioria dos trabalhos que retratam sobre a taxa de mortalidade nesses estados brasileiros são antigos e necessitam de levantamento mais detalhado a respeito dessa doença no país.

Com isso o governo brasileiro deve investir financeiramente com o intuito de incentivar a pesquisa cientifica a fim de obter estudos mais aprofundados a respeito da mortalidade por essa doença neurológica, visto que no Brasil ela acomete milhares de pessoas que necessitam de tratamentos específicos, como farmacológico e o psicológico (Chaves et al., 2018). Ainda não existe cura definitiva para essa doença. O tratamento se dá por meio de drogas farmacológicas como, por exemplo, Ziledon e Donezepila; contudo, estudos científicos estão sendo desenvolvidos com células-tronco e nanofármacos, considerados candidatos promissores para a patogêneses do Alzheimer (Srivastava et al., 2021).

De acordo com a Figura 1, houve um aumento na taxa de mortalidade no país durante o período de estudo, ou seja, o número de mortes por Alzheimer vem crescendo de forma exponencial com o passar dos anos. Em 2010 o número de mortes era 10.841 e em 2019 esse número aumentou para 23.150. Que corresponde a um aumento de 133,54\%. Do mesmo modo, a taxa de mortalidade para o ano de 2010 foi de 5,68 mortes por 100.000 habitantes e em 2019 foi de 11,01/100.000 habitantes, sendo constado um aumento percentual de $93.81 \%$. 
No Brasil os pacientes com Alzheimer representam diferenças demográficas importantes, em comparação com os pacientes que não possuem essa doença, pois dois em cada cinco brasileiros se sentem solitários, e em cada três se sentem deprimidos, ou até mesmo triste na maioria do tempo (Feter et al., 2021). No país, as políticas públicas voltadas para essa doença ainda são escassas, possuindo lacunas a respeito de suas causas e tratamento, os estudos científicos são mais voltados para a doença retratam que ainda necessitam de maiores investimento (Costa et al., 2021).

O cuidado e o exercício empregados às pessoas com Alzheimer sempre será uma questão de saúde pública, tendo como dever, o estado, de melhorar as estratégias e abordagens do tratamento dessa doença a fim de diminuir os impactos causados às pessoas. Uma forma de diminuir tais impactos é através da disponibilidade, de forma acessível, dos medicamentos voltados para essa doença, pois diversos medicamentos são caros e muitas vezes inacessíveis através do Sistema Único de Saúde (SUS) (Soares et al., 2017; Barbosa et al., 2020).

Figura 1. Taxa de mortalidade anual e número absoluto de óbitos relacionado à Alzheimer durante os anos de 2010 a 2019 no Brasil.

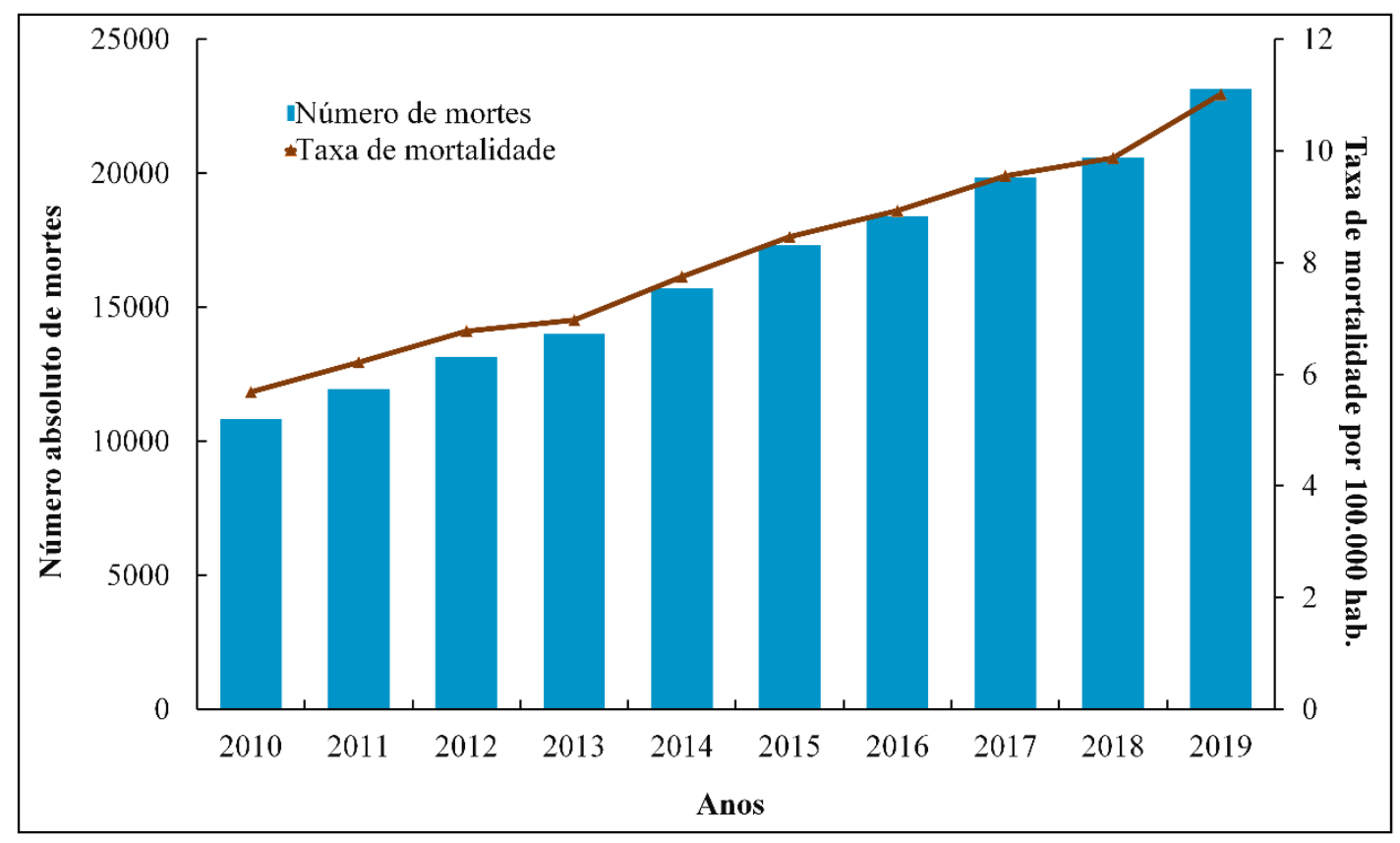

Fonte: Autores.

Em relação às características sociodemográficas predominantes dos casos foram: o sexo feminino $(64,54 \%)$, faixa etária $\geq 80(74,99 \%)$; pessoas brancas $(73,80 \%)$ e pessoas viúvas $(50,66 \%)$, no entanto, alguns dados foram ignorados $(9,42 \%)$, em que não se sabe o gênero, a faixa etária, a raça e o estado civil de alguns pacientes (Tabela 2).

Segundo Fernandes et al (2018), retrata que o Alzheimer é uma demência cerebral primária que tem associação com o sexo e a idade onde sua prevalência é entre mulheres idosas. Notou-se que essas mulheres sofreram diversas alterações durante sua vida, comprometendo suas atividades diárias, causando angústia e dependência pelo cuidador. 
Tabela 2. Características sociodemográficas dos óbitos relacionados às Alzheimer no Brasil entre 2010 a 2019.

\begin{tabular}{lll}
\hline Variáveis & $\mathbf{N}^{\mathbf{0}}$ Absoluto de Casos & Frequência (\%) \\
\hline Gênero & & \\
Masculino & 58.473 & 35,44 \\
Feminino & 106.485 & 64,54 \\
Ignorados & 18 & 0,01
\end{tabular}

\section{Faixa etária}

\begin{tabular}{lll}
$0-19$ & 0 & 0 \\
$20-29$ & 3 & 0,01 \\
$30-39$ & 4 & 0,002 \\
$40-49$ & 103 & 0,06 \\
$50-59$ & 694 & 0,42 \\
$60-69$ & 6.396 & 3,87 \\
$70-79$ & 34.034 & 20,62 \\
$\geq 80$ & 123.728 & 74,99 \\
Ignorado & 14 & 0,008 \\
& & \\
Raça/Cor & & \\
Branco & 121.754 & 73,80 \\
Preta & 6.450 & 3,90 \\
Amarelo & 1.450 & 0,87 \\
Parda & 29.450 & 17,85 \\
Indígena & 85 & 0,05 \\
Ignorado & 5.787 & 3,50 \\
& & \\
Estado civil & & 9,95 \\
Solteiro & 16.422 & 28,98 \\
Casado & 47.822 & 50,66 \\
Viúvo & 83.591 & 3,81 \\
Separado judicialmente & 6.297 & \\
Outro & 1.118 & \\
Ignorado & 9.726 & \\
\hline
\end{tabular}

Fonte: Autores.

A doença de Alzheimer acaba se tornando sintomática quando as lesões cerebrais conseguem atingir determinado limiar dentro da suscetibilidade única do paciente em que fatores genéticos e epigenéticos estão associados à doença (Dubois et al., 2016). Na literatura cientifica existem poucos trabalhos a respeito da epidemiologia e dos aspectos sociodemográficos do Alzheimer nos estados brasileiros ou municípios. Porém o estudo de Teixeira e Filha (2014) retrata que na cidade Porto Alegre, pertencente ao estado do Rio Grande do Sul, nos anos de 2000 a 2009 a taxa de mortalidade no município era predominante em homens.

Segundo o trabalho de Oliveira et al (2015), os dados sociodemográficos a respeito da mortalidade apontam que pessoas pardas, do sexo masculino, caracterizam o grupo social de maior vulnerabilidade na faixa etária de idosos, mas se deve levar em consideração que os jovens não estão isentos dessa doença neurológica. Um estudo realizado nos Estados Unidos por Seifi e colaboradores (2021), retrata que jovens hispânicos apresentaram maior prevalência da doença.

Segundo a Organização de Saúde (2012), é retratada a necessidade de políticas públicas mais eficientes que apontem e permitam incremento na assistência da pessoa idosa por meio de estratégias implementadas pelo ministério da Saúde e outras instituições do governo brasileiro (Ferreira et al., 2016). Os determinantes demográficos, culturais e econômicos tornam a 
questão dessas pessoas de extrema importância para a sociedade, em que as políticas exclusivamente dirigidas para este segmento etário devem ser concretizadas urgentemente (Rocha \& Bartholo, 2010).

Tendo em conta que quem realiza esses cuidados são, em sua maioria, pessoas do sexo feminino e geralmente filhas ou esposas do paciente com Alzheimer, com idade acima de 60 anos; ou seja, uma pessoa idosa cuidando de outra idosa, por conta disso o estado necessita investir em políticas que auxiliem essas pessoas no cuidado (Dadalto \& Cavalcante, 2021).

Outra questão a ser discutida é o estresse ocasionado por essa doença ao cuidador, seja da família ou até mesmo contratado, como por exemplo um técnico em enfermagem, pois a doença demanda tempo e capacidade de lidar com as dificuldades diárias, com em casos de alterações de humor do paciente, onde o cuidador fica sobrecarregado e acaba se esquecendo de cuidar de si mesmo, podendo gerar mal-estar e prejudicar sua saúde emocional e mental (Risso et al., 2021).

\section{Considerações Finais}

Diante desse estudo foi verificado que os maiores percentuais em relação às variáveis sociodemográficas se concentraram no sexo feminino de faixa etária a partir de 80 anos, brancas e com estado civil viúvo (a). É de extrema importância uma supervisão relacionada às políticas públicas voltadas para esse assunto, em que as pessoas idosas do sexo feminino são as mais vulneráveis a essa doença neurodegenerativa. Com isso recomenda-se que os profissionais na área da saúde desenvolvam métodos de notificação mais eficazes que consigam suprir essa deficiência, aprimorando a qualidade de informação dos dados voltados para saúde. Dessa forma, esse trabalho enfatiza a necessidade de investimentos em saúde pública, pois só assim os pacientes que possuem a doença de Alzheimer poderão ter atenção ao atendimento e adesão ao tratamento referente a essa doença.

\section{Referências}

Barbosa, M. G. A et al (2020) O uso do composto de Canabidiol no tratamento da doença de Alzheimer (revisão da literatura). Research, Society and Development, 9(8), e442986073-e442986073.

Chaves, J. C et al (2018) Tratamento farmacológico e assistência psicológica na Doença de Alzheimer. Revista Saúde em Foco.

Costa, C. F. P et al (2021) Políticas públicas preventivas voltadas ao estado demencial causado pela doença de Alzheimer. Revista CPAQV-Centro de Pesquisas Avançadas em Qualidade de Vida-CPAQV Journal, 13 (1).

Dadalto, E. V. \& Cavalcante, F. G. (2021) O lugar do cuidador familiar de idosos com doença de Alzheimer: uma revisão de literatura no Brasil e Estados Unidos. Ciência \& Saúde Coletiva, 26, 147-157.

Dubois, B et al (2016) Preclinical Alzheimer's disease: definition, natural history, and diagnostic criteria. Alzheimer's \& Dementia, 12 (3), $292-323$.

Favaretto, B. G. S et al (2021) Epidemiologia de demência no SUS: O exemplo da doença de Alzheimer no Tocantins. Revista de Patologia do Tocantins, 8 (1).

Ferreira, A. B. T et al (2016) Mortalidade pela Doença de Alzheimer no Brasil Entre 2000 a 2013. Acta de Ciências e Saúde, 1 (1), 1-16.

Fernandes, M. R. S et al (2018) Doença de Alzheimer nas Mulheres: prejuízos pessoais e luto familiar. Id on Line Revista De Psicologia, 12 (39), 533-551.

Feter, N et al (2021) Quem são as pessoas com Doença de Alzheimer no Brasil? Resultados do Estudo Longitudinal da Saúde dos Idosos Brasileiros (ELSIBrasil). Revista Brasileira de Epidemiologia.

IBGE. Instituto Brasileiro de Geografia e Estatística. Brasil. 2020. https://www.ibge.gov.br/cidades-e-estados.html.

Long, J. M. \& Holtzman, D. M. (2019) Alzheimer disease: an update on pathobiology and treatment strategies. Cell, 179 (2), $312-339$.

Magalhães, T et al (2020) Aspectos clínicos e sociais dos portadores da doença de Alzheimer na cidade de Muritiba - BA. Enciclopédia Biosfera, 17 (33).

Oliveira, T. C et al (2015) Diferenciais sócio-demográficos da mortalidade de idosos em idades precoces e longevas. Revista Baiana de Saúde Pública, 39 (2), 249-249.

Pereira, F. Z et al (2021) Mortalidade Infantil e sua relação com as políticas públicas em saúde sob o olhar dos Objetivos de Desenvolvimento do Milênio e Objetivos de Desenvolvimento Sustentável no Estado de Goiás. Brazilian Journal of Health Review, 4 (1), 3331 -3348. 
Research, Society and Development, v. 10, n. 11, e74101119316, 2021

(CC BY 4.0) | ISSN 2525-3409 | DOI: http://dx.doi.org/10.33448/rsd-v10i11.19316

Risso, D. T et al (2021) Avaliação das dificuldades e sobrecarga do cuidador de pessoas com a doença de Alzheimer. Brazilian Journal of Development, 7 (5), 52004-52012.

Rocha, F. N. \& Bartholo. M. C. (2010) Educação e Qualidade de Vida de Idosos: Uma Reflexão Necessária. http://editora.universidadedevassouras.edu.br/index.php/RM/article/view/121.

Santos, D. M et al (2019) Morbidade e mortalidade da doença de Alzheimer em indivíduos hospitalizados no Brasil, entre 2008 e 2018 : estudo ecológico. Revista de Ciências Médicas e Biológicas, 18 (3), 314-319.

Seifi, A et al (2021) Hispanic Ethnicity and In-hospital Morbidity and Mortality Outcomes in Alzheimer's Disease: A US National Study 2005-2015. Clinical Neurology and Neurosurgery.

Soares, N. M et al (2017) Impacto econômico e prevalência da doença de Alzheimer em uma capital Brasileira. Ciência \& Saúde, 10 (3), 133-138.

Srivastava, S et al (2021) Alzheimer's disease and its treatment by different approaches: a review. European Journal of Medicinal Chemistry.

Teixeira, J et al (2012) H. P2-159: Profile of mortality from Alzheimer's disease: Rio de Janeiro 2000-2006. Alzheimer's \& Dementia, 8 (4), 314-314.

Teixeira, J. B. \& Filha, M. M. T. (2014) Alzheimer's Disease: A study of mortality in the cities of Porto Alegre And Vitória (Brazil). (2014) Alzheimer's \& Dementia: The Journal of the Alzheimer's Association, 4 (10), 911.

Vidor, R. C et al (2019) Mortalidade por doença de Alzheimer e desenvolvimento humano no século XXI: um estudo ecológico nas grandes regiões brasileiras. ACM arq. catarin. med, 94-107.

World Health Organization. Dementia: a public health priority. World Health Organization, 2012. https://www.who.int/publications/i/item/dementia-a-publichealth-priority.

Yang, S. J., Shin, H, Lee, S. H. \& Lee, S. K. (2020). Functional linear regression model with randomly censored data: Predicting conversion time to Alzheimer's disease. Computational Statistics \& Data Analysis, 150, 107009. 